\title{
ANALIZANDO LA EVOLUCIÓN DEL MODELADO DE ENFERMEDADES INFECCIOSAS
}

\author{
ANALISYNG THE EVOLUTION OF INFECTIOUS \\ DISEASES MODELLING
}

\author{
Félix Sebastián Rincón Tobo ${ }^{1}$ \\ Javier Antonio Ballesteros-Ricaurte ${ }^{2}$ \\ Ángela María González Amarillo ${ }^{3}$
}

\begin{abstract}
1. Maestría en Tecnología Informática, Universidad Pedagógica y Tecnológica de Colombia, Tunja, Boyacá, Colombia.
${ }^{2}$ PhD(c) en ingeniería, Universidad Pedagógica y Tecnológica de Colombia, Tunja, Boyacá, Colombia.
\end{abstract}

${ }_{3}^{3}$ Maestría en Administración de Empresas, Universidad Nacional Abierta y a Distancia, Tunja, Boyacá, Colombia.

\footnotetext{
${ }^{1}$ felix.rincon@uptc.edu.co

2 javier.ballesteros@uptc.edu.co

3 angela.gonzalez@unad.edu.co
}

\section{RESUMEN}

El interés global por conocer y controlar las enfermedades que afectan a humanos y animales ha permitido modelar enfermedades mediante diversos métodos (modelos matemáticos, estocásticos, discretos) que se aplican actualmente para predecir la propagación de nuevas epidemias, reducir el contagio de enfermedades infecciosas, evaluar el impacto que tendrán las diferentes estrategias de control de enfermedades y mejorar las condiciones de vida de los individuos. Actualmente, nuevas técnicas y herramientas se están implementando para modelar enfermedades infecciosas, el presente documento describe conceptos de esta área, así como las tendencias y retos existentes, finalmente se ofrecen al lector algunos criterios

a considerar para la selección de un modelo epidemiológico.

Palabras clave: Control de epidemias; enfermedades infecciosas; impacto; modelo epidemiológico.

\section{ABSTRACT}

The global interest to know and deal with infectious diseases in humans and animals has led to the development of different models (mathematical, stochastic, discrete), applied to predict the spread of new epidemics, reduce the spread of infectious diseases, evaluate the impact of different disease control strategies and improve the living conditions of individuals. Nowadays, new techniques and tools 
are being implemented to model infectious diseases, this paper describes the main concepts of this area, current trends and existing challenges, and finally, describes some criteria for the selection of an epidemiological model.

Key words: infectious diseases, epidemiological model, impact, epidemic control.

\section{Resumen gráfico}

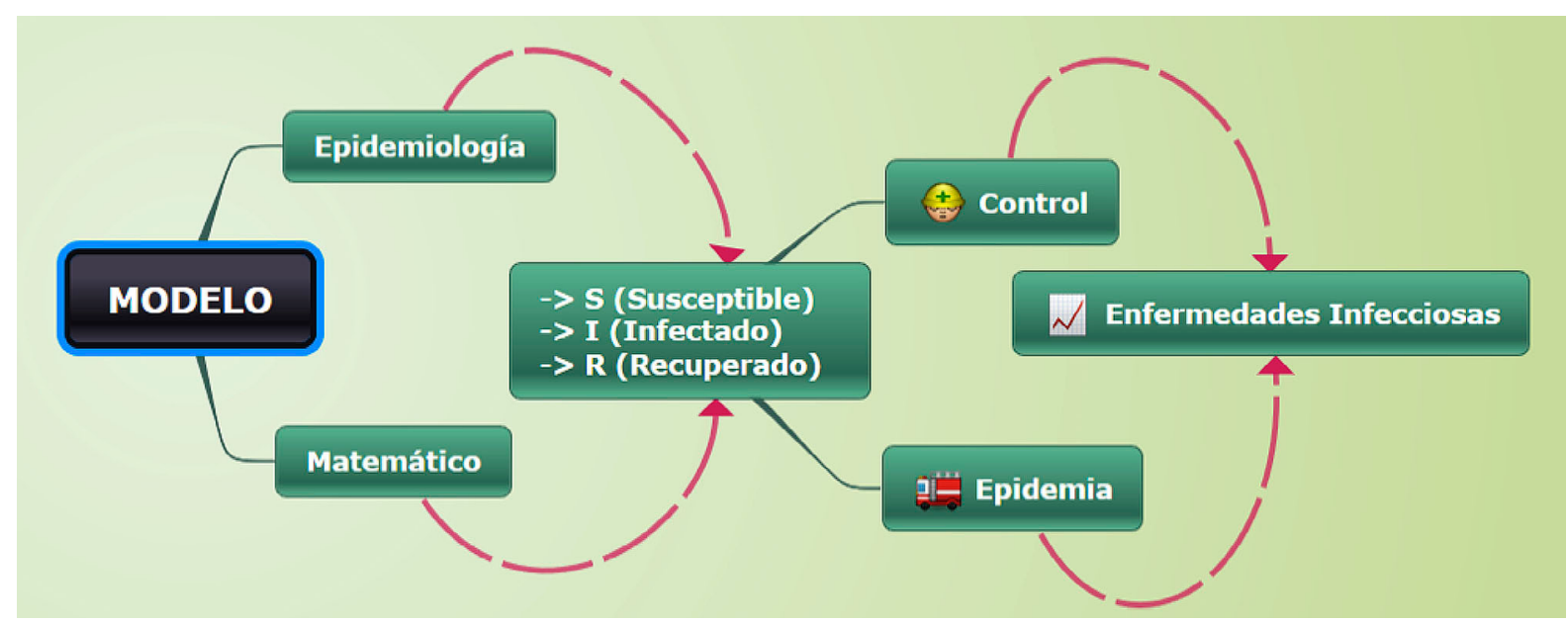

\section{INTRODUCCIÓN}

Hay un vertiginoso aumento en la capacidad de computo disponible, apalancando el desarrollo técnico y científico en diferentes áreas del conocimiento (Pérez, 2010), dentro de las que se encuentra la epidemiología, la cual busca comprender la distribución, incidencia y control de enfermedades.

Los primeros esfuerzos humanos por comprender el comportamiento de las enfermedades datan de la antigua Grecia en los tratados hipocráticos (University of Chicago, 2016); luego, en 1760 Daniel Bernoulli plantea uno de los primeros modelos matemáticos que simula el comportamiento de una enfermedad (Pedersen, 2015). Sin embargo, esta tendencia solo toma fuerza hasta principios del siglo $X X$, momento en que se empieza a dar relevancia a la epidemiologia, al modelado de enfermedades y se incrementa la generación de textos científicos en el área (Hernández, 2007).
Un modelo epidemiológico es una representación del comportamiento de una enfermedad en una población, ya sea de humanos, plantas o animales, con el objetivo de predecir la rapidez de contagio de nuevos individuos, qué va a pasar con la epidemia en un futuro y qué efecto tendrán las estrategias de control como vacunación, cuarentena o sacrificio (Keeling \& Rohani, 2011). Actualmente, los modelos epidemiológicos tienen diversas aplicaciones en diversas áreas, por citar algunos ejemplos se tienen: modelos geoespaciales de enfermedades infecciosas en fauna silvestre (Ligarte, Goicoa, Ibáñez \& Militino, 2009; Staubach, Schmid, Knorr-Held \& Ziller \& 2002), el modelado epidemiológico y económico de la influenza aviar para evaluar estrategias de control de la enfermedad (Backer, van Roermund, Fischer, van Asseldonk \& Bergevoet, 2015), el modelo epidemiológico para comprender el contagio de la carne de cerdo de salmonela en 
el proceso de sacrificio (Savall, Bidot, LeblancMaridor, Belloc \& Touzeau, 2016), el modelo epidemiológico para agrupar tendencias en publicaciones científicas (Marutschke \& Ogawa, 2014); y adicionalmente, los modelos epidemiológicos han sido estudiados para generar nuevos modelos teniendo en cuenta la relación de redes sociales y redes de computadores, donde hay nuevas variables en contextos diferentes (Sahneh, Vajdi, Melander \& Scoglio, 2017). Si bien el campo de aplicación de los modelos epidemiológicos es muy amplio, estos presentan algunos inconvenientes, pues muchos de los factores que deben ser tenidos en cuenta para comprender una enfermedad son de naturaleza aleatoria y no lineal, además las simplificaciones que realizan los modelos epidemiológicos rara vez contemplan la situación de los individuos de forma individual.

El reto actual para los investigadores en el área radica en superar estas dificultades y proponer nuevos modelos que abarquen el carácter aleatorio y no lineal de las epidemias, así como avanzar en la comprensión de los fenómenos epidemiológicos desde el punto de vista de los individuos de forma particular.

El presente artículo introduce al lector en el campo de los modelos epidemiológicos mostrando las bases analíticas y teóricas necesarias para el desarrollo de dicha actividad, posteriormente clasifica y analiza artículos científicos desarrollados en esta área que muestran el alcance y enfoque que tienen estos modelos, finalmente sugiere algunos aspectos y elementos que debe tener en cuenta el autor para la elección de un modelo epidemiológico.

\section{ANTECEDENTES}

\section{A. Primer Modelo}

Daniel Bernoulli realizó el primer acercamiento al proceso de modelado de una enfermedad, su objetivo era determinar la conveniencia de utilizar una vacuna contra la viruela aun cuando esta no era totalmente efectiva (Pedersen, 2015), para tal fin Bernoulli realizó los siguientes supuestos (Bacaër, 2011):

- Las personas que contraen viruela por primera vez mueren con una probabilidad $\boldsymbol{p}$ y sobreviven con una probabilidad $(\mathbf{1}-\boldsymbol{p})$.

- Cualquier persona tiene una probabilidad $\boldsymbol{q}$ de ser infectado cada año, por tanto, la probabilidad que tiene un individuo de ser infectado entre la edad $x$ y la edad $\boldsymbol{x}+\boldsymbol{d x}$ es $\boldsymbol{q} . \boldsymbol{d} \boldsymbol{x}$ siendo $\mathrm{dx}$ un periodo infinitesimal de tiempo.

- Las personas que son infectadas con viruela y sobreviven adquieren inmunidad de por vida.

- Sí $\boldsymbol{m}(\boldsymbol{x})$ es la probabilidad de morir a una edad $\boldsymbol{x}$ por causas diferentes a la viruela, la probabilidad de un individuo de morir en un periodo de tiempo infinitesimal entre la edad $\boldsymbol{x}$ y la edad $\boldsymbol{x}+\boldsymbol{d} \boldsymbol{x}$ es $\boldsymbol{m}(\boldsymbol{x}) \cdot \boldsymbol{d} \boldsymbol{x}$.

- El número de personas nacidas en un mismo año se denota como $\boldsymbol{P}_{\boldsymbol{o}}$.

- $\boldsymbol{S}(\boldsymbol{x})$ es el número de personas aún vivas con edad $\boldsymbol{x}$ que nunca han contraído viruela.

- $\boldsymbol{R}(\boldsymbol{x})$ es el número de personas aún vivas con edad $\boldsymbol{x}$ que contrajeron viruela y sobrevivieron.

- $\boldsymbol{P}(\boldsymbol{x})=\boldsymbol{S}(\boldsymbol{x})+\boldsymbol{R}(\boldsymbol{x})$, siendo $\boldsymbol{P}(\boldsymbol{x})$ el número total de personas vivas a la edad $\boldsymbol{x}$.

Para simplificar el modelo se asume que no nacen nuevas personas.

De acuerdo con las observaciones de Bernoulli y al uso que él realizó de las tablas de Halley, la probabilidad de morir por viruela $\boldsymbol{p}$ es igual a $\mathbf{1 / 8}$ y la probabilidad de contraer viruela $\boldsymbol{q}$ es igual a 1/8, considerando que la vacuna es totalmente efectiva y se ha aplicada a todos los individuos al nacer conlleva al incremento de la esperanza de vida, siendo $\boldsymbol{P} *(\boldsymbol{x})$ la población de edad $x$ viva en un periodo de tiempo $d x$. 
De aquí se deduce que con una vacunación efectiva la esperanza de vida de la población aumenta 3.38 años, sin embargo, la vacunación no era completamente segura, por tanto, si $\boldsymbol{p}^{\prime}$ es la probabilidad que tiene un individuo de morir por viruela luego de ser inoculado, pero teniendo en cuenta que $\boldsymbol{p}^{\prime}<\boldsymbol{p}$. La esperanza de vida sería $\left(\mathbf{1}-\boldsymbol{p}^{\prime}\right)$. $\boldsymbol{E}^{*}$ que seguiría siendo mayor que sin vacunación siempre $y$ cuando $\boldsymbol{p}^{\prime}<\mathbf{1}-\boldsymbol{E} / \boldsymbol{E}^{*}$ o $\boldsymbol{p}^{\prime}<11 \%$, Bernoulli estimo que la probabilidad de morir luego de ser inoculado $\boldsymbol{p}^{\prime}$ era cerca del $1 \%$ así que, aun cuando la inoculación no fuese $100 \%$ efectiva, esta debía ser promovida y aplicada.

Con el aporte de Daniel Bernoulli se crea un precedente de la manera en que las herramientas matemáticas permiten modelar y mejorar la comprensión de los fenómenos epidemiológicos, aportando así al bienestar de los individuos.

\section{Aproximación al Modelo SIR}

En 1927, William Kermack y Anderson Mckendrick presentan en donde se define lo que hoy en día se conoce como un modelo SIR (Susceptible, Infectado, Recuperado) y que en la actualidad sigue siendo la base para un gran número de modelos epidemiológicos (Kermarck \& McKendrick, 1927).

El modelo planteado, difiere del que planteó Daniel Bernoulli, puesto que para él solo existían los estados S (susceptible) y R (recuperado), mientras que en este caso existen 3 estados: el estado $X$ en el que se encuentran los individuos susceptibles, el estado $Y$ en el que se encuentran las personas infectadas $y$ el estado $Z$ donde se encuentran los individuos recuperados. Luego de realizar consideraciones, como asumir la inexistencia de muertes y de nacimientos, y la obtención de inmunidad de por vida tras haberse recuperado de la enfermedad, Kermack y McKendrick llegan a las ecuaciones 1 a 3 que definen los 3 estados del modelo (Kermack \& McKendrick, 1927).

$$
\begin{array}{ll}
\frac{d x}{d t}=-k \cdot x \cdot y & \text { (Ecuación 1) } \\
\frac{d y}{d t}=k \cdot x \cdot y-l \cdot y & \text { (Ecuación 2) } \\
\frac{d z}{d t}=l \cdot y & \text { (Ecuación 3) }
\end{array}
$$

Donde $\boldsymbol{d} \boldsymbol{x} / \boldsymbol{d} \boldsymbol{t}$ es la variación de personas en estado susceptible, $\boldsymbol{k}$ es la razón a la que se enferman nuevos individuos, $\boldsymbol{x}$ es el número de individuos susceptibles, $\boldsymbol{y}$ es el número de individuos infectados y l es la razón a la que se recuperan los individuos infectados.

A partir de este modelo se han realizado numerosas variaciones de acuerdo con las características de cada enfermedad, agregando o suprimiendo estados, considerando muertes y nacimientos y generando herramientas para determinar con mayor precisión las tasas de contagio y recuperación.

\section{Modelo Epidemiológico SIR}

El modelo SIR (figura 1) está pensado para ser aplicado en enfermedades infecciosas ignorando la demografía (nacimientos, muertes y migraciones), en este modelo existen 3 estados en los que puede encontrarse un individuo (Susceptible, Infectado, Recuperado) y solo existen 2 transiciones posibles (Susceptible -> Infectado e Infectado -> Recuperado). La transición de S->I es producto de un proceso infeccioso en el que intervienen factores como: la prevalencia de la enfermedad, el contacto entre individuos susceptibles e infectados y la probabilidad de contagio debido un contacto; mientras que la transición entre Infectados y Recuperados está dada por la prevalencia de la enfermedad y las características de la misma que ocasionan una tasa de recuperación particulares para cada enfermedad. 


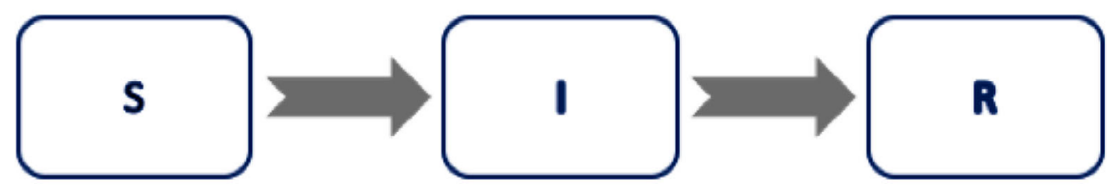

Figura 1. Diagrama de flujo Modelo SIR.

Fuente: Tomado de (Kermarck \& McKendrick, 1927)

Para determinar el funcionamiento de este modelo y de acuerdo con lo expuesto por Keeling y Rohani (Keeling \& Rohani, 2011), es necesario definir algunos factores:

- $\boldsymbol{\lambda}$ es la fuerza de infección y es la tasa per cápita a la que los individuos susceptibles contraen la enfermedad

- $\boldsymbol{X}$ es el número de individuos en el estado $\mathbf{S}$

- $\boldsymbol{\lambda} . \boldsymbol{X}$ es la tasa a la que se producen nuevos infectados

- $\boldsymbol{\lambda}$ es proporcional al número de individuos infectados

- Existen dos enfoques para calcular $\lambda$, el primero depende de la frecuencia de contacto entre individuos susceptibles e infectados y está dado por $\boldsymbol{\lambda}=\boldsymbol{\beta} . \boldsymbol{Y} / \boldsymbol{N}$ y un segundo enfoque en el que $\boldsymbol{\lambda}$ depende de la densidad de población siendo $\boldsymbol{\lambda}=\boldsymbol{\beta}$. $Y$ donde $\boldsymbol{Y}$ es el número de individuos en el estado $\mathbf{I} ; \boldsymbol{N}$ es el total de la población; $\beta$ es el producto de la tasa de contacto entre individuos y la probabilidad de contagio dado un contacto.

- El enfoque de frecuencia de contacto refleja las situaciones en las que la probabilidad de contagio no depende del tamaño de la población, es decir no existen mayores posibilidades de contraer gripa en una ciudad de 1 millón de habitantes que en una de 100.000 habitantes. Keeling \& Rohani (2011) identifican que este enfoque es más adecuado para modelar enfermedades transmitidas por vectores que tienen estructuras de contacto heterogéneas.

- El enfoque basado en la densidad se fundamenta en el hecho que entre más grande sea una población, mayor número de contactos aleatorios van a existir entre un individuo sano y uno susceptible, este enfoque es mayormente aplicado al modelado de enfermedades en plantas y animales.

- Para simplificar la notación es conveniente usar $\boldsymbol{S}=(\boldsymbol{X} / \boldsymbol{N}), \boldsymbol{I}=(\boldsymbol{Y} / \boldsymbol{N})$ donde $\boldsymbol{I}$ y $\boldsymbol{S}$ son la proporción de individuos infectados y susceptibles, respectivamente.

- Retomando la tasa de nuevos contagios dada por $\boldsymbol{\lambda} . \boldsymbol{X}$, se tiene la ecuación 4 .

\section{$\boldsymbol{\lambda} \cdot \boldsymbol{X}=\boldsymbol{\beta} . \boldsymbol{I} \cdot \boldsymbol{X} \quad$ (Ecuación 4)}

Con el objetivo de simplificar la notación y tener la ecuación en términos de densidades de población $\boldsymbol{I}$ y $\boldsymbol{S}$, se remplaza $\boldsymbol{S}=\boldsymbol{X} / \boldsymbol{N}$, obteniendo la ecuación 5 .

$$
\frac{d S}{d t}=-\beta . I . S \quad(\text { Ecuación 5) }
$$

Para determinar la variación en la densidad de población infectada $\boldsymbol{I}$ se debe considerar la transición que se produce desde el estado $\mathrm{S}$, que sería la misma mostrada en la ecuación 14, pero con signo contrario, y la transición 
que va desde $\boldsymbol{I}$ hacia $\boldsymbol{R}$, que depende de la densidad de población infectada y de la tasa de recuperación que para este caso se denota $Y$, con estas consideraciones se obtiene la ecuación 6 ( Keeling \& Rohani, 2011).

$$
\frac{d I}{d t}=\beta . I . S-\gamma . I \quad(\text { Ecuación 6) }
$$

Ahora, la variación de densidad de población Recuperada, depende únicamente de la transición proveniente del estado I, ya que previamente se asumió que no existían muertes, nacimientos, ni la posibilidad de ser susceptible nuevamente; de tal forma que la ecuación 7 (Keeling \& Rohani, 2011) describe la variación del estado R.

$$
\frac{d R}{d t}=\gamma \cdot I \quad(\text { Ecuación 7) }
$$

De esta forma se tienen las ecuaciones 5, 6 y 7 que describen el sistema SIR, adicionalmente es necesario describir algunos parámetros que son de interés y que permiten determinar con facilidad el comportamiento que tendrá el modelo:

\section{Tasa de recuperación}

La tasa de recuperación $\boldsymbol{V}$ en las ecuaciones previas, es velocidad a la que se recuperan los individuos infectados, sin embargo, su inverso $1 / \boldsymbol{V}=\boldsymbol{T}$ es el tiempo promedio que dura enfermo un individuo, dato que puede ser calculado fácilmente a partir de los registros médicos.

\section{El fenómeno del Umbral}

Al considerar la condición inicial de un modelo en el que no hay ningún individuo infectado ni recuperado, todos los individuos se encuentran susceptibles el fenómeno de introducir un individuo infectado permite identificar los factores que definen si ocurrirá una epidemia, o solo unos pocos individuos se infectaran antes de que la epidemia desaparezca. Reescribiendo la ecuación 6 se obtiene 8 ( Keeling \& Rohani, 2011).

$$
\frac{d I}{d t}=I(\beta . S-\gamma) \quad(\text { Ecuación 8) }
$$

Despejando S de la desigualdad se obtiene la ecuación 9.

$$
\frac{\gamma}{\boldsymbol{\beta}}<\boldsymbol{S} \quad \text { (Ecuación 9) }
$$

De manera que para que $\boldsymbol{d} \mathbf{l} / \boldsymbol{d} \boldsymbol{t}$ sea positivo $\mathrm{y}$ se produzca una epidemia, $\mathrm{S}(0)$ debe ser mayor que $\boldsymbol{V} / \boldsymbol{\beta}$ fracción que es denominada Tasa relativa de recuperación y su inversa se denomina Tasa básica de reproducción o RO. Dadas estas consideraciones y teniendo en cuenta que $S(0)$ se concluye que:

En la tabla 1, se muestra la estimación de algunas tasas básicas de reproducción. 
Tabla 1. Tasas básicas de reproducción.

\begin{tabular}{l|c|c|c}
\hline Infectious Disease & Host & Estimated Ro & Reference \\
\hline FIV & Domestic cats & $1.1-1.5$ & Smith (2001) \\
\hline Rabies & Dogs (Kenya) & 2.44 & Kitala et al. (2002) \\
\hline Phocine Distemper & Seals & $2-3$ & Swinton et al. (1998) \\
\hline Tuberculosis & Cattle & 2.6 & Goodchild and Clifton-Hadley (2001) \\
\hline Influenza & Humans & $3-4$ & Murray (1989) \\
\hline Food-and-Mouth Disease & Livestock farms (UK) & $3.5-4.5$ & Ferguson et al. (2001b) \\
\hline Smallpox & Humans & $3.5-6$ & Gani and Leach (2001) \\
\hline Rubella & Humans (UK) & $6-7$ & Anderson and May (1991) \\
\hline Chickenpox & Humans (UK) & $10-12$ & Anderson and May (1991) \\
\hline Measles & Humans (UK) & $16-18$ & Anderson and May (1982) \\
\hline Whooping Cough & Humans (UK) & $16-18$ & Anderson and May (1982) \\
\hline
\end{tabular}

Fuente: Tomado de (Keeling \& Rohani, 2011)

\section{TENDENCIA ACTUAL}

A partir de aportes representativos como el de Bernoulli (1760) y el de Kermack y McKendrick (1927) se establecen las bases para el desarrollo de modelos epidemiológicos muy diversos, cuyo objetivo puede clasificarse de acuerdo con dos variables: finalidad y tipo de modelo, donde la finalidad puede ser el análisis y proyección de epidemias o la evaluación de estrategias de control; mientras que el tipo de modelo se refieren al uso de modelos epidemiológicos existentes o a la creación de nuevos modelos; considerando estas dos variables y analizando 52 artículos de modelos epidemiológicos publicados desde el año 2013 en las bases de datos bibliográficos Science Direct y Scopus se obtiene la figura 2 que cuenta con 4 posibles categorías producto del cruce de las dos variables planteadas:

1) uso de modelos existentes para evaluar estrategias de control de epidemias,

2) uso de modelos existentes para el análisis de nuevas enfermedades infecciosas o de enfermedades infecciosas ya modeladas, pero con características particulares,

3) la generación de nuevos modelos para evaluar estrategias de control de epidemias, y,

4) la formulación de nuevos modelos para describir de forma más precisa enfermedades previamente modeladas o enfermedades en condiciones particulares o nuevas enfermedades.

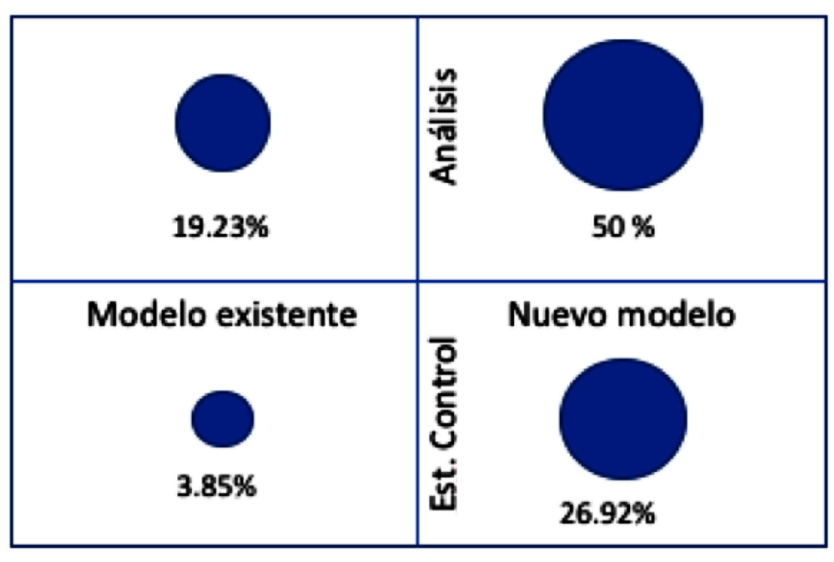

Figura 2. Clasificación de artículos de modelos epidemiológicos.

Fuente: Autores. 


\section{Estrategias de control de epidemias mediante modelos existentes.}

El análisis de estrategias de control de epidemias mediante modelos existentes solo representa un $3.85 \%$ (figura 2 ) de la literatura revisada, este tipo de investigaciones se realizan cuando es necesario definir la efectividad de una estrategia de control para una enfermedad bien descrita en un entorno específico, así que se utiliza un modelo existente pero ajustando las condiciones locales, por ejemplo, se ha realizado el análisis del impacto del rotavirus con un modelo SVIR (susceptible, vacunado, infectado, recuperado) ajustándolo mediante el uso parámetros como la rata de nacimientos y el tamaño de la población propios de Gales e Inglaterra (Atkins, Shim, Pitzer \& Galvin, 2012).

También es posible realizar investigaciones en esta área para evaluar nuevos métodos de control de enfermedades que permiten ser descritas por modelos básicos, Zaman, Kang \& Jung (2009) aplican la teoría de control óptima para mejorar estrategias en un modelo tipo SIR (susceptible, infectado, recuperado) con retardo y se regula la propagación de la enfermedad modelada. Sin embargo, el bajo número de investigaciones realizadas muestran una tendencia a plantear nuevos modelos que describan con mayor precisión el comportamiento de las enfermedades y consideren fenómenos adicionales a los descritos en modelos epidemiológicos básicos (Gaucel, Laroche, Ezanno \& Touzeau, 2009; Van Effelterre, Marano \& Jacobsen, 2016) como lo son la interacción entre individuos y comunidades (Gaucel, Laroche, Ezanno \& Touzeau, 2009; Ssematimba, Jores \& Mariner, 2015; Bekara, Courcoul, Bénet \& Durand, 2014; Cai, Wang \& Wang, 2016; Rossi, De Leo, Pongolini, Natalini, Vincenzi \& Bolzoni, 2015) o los desplazamientos y migraciones (Fan, Zhang \& Gao, 2016; Njagarah \& Nyabadza, 2014; Rao, 2016).

\section{Estrategias de Control de Epidemias Mediante Nuevos Modelos}

Muchos de los fenómenos que pueden ser descritos mediante modelos epidemiológicos tienen un alto grado de complejidad, si bien, la idea al producir un modelo es simplificar el fenómeno, existe un compromiso entre la simplicidad y la exactitud, siendo determinante la finalidad del modelo para definir su nivel de complejidad. Cuando la finalidad es evaluar o definir métodos de control, es muy importante la exactitud, ya que solo es posible controlar aquello que se conoce. En la revisión de los artículos reportados en las bases de datos previamente descritas se encontró que $26.92 \%$ (figura 2) de los documentos plantean nuevos modelos epidemiológicos para evaluar estrategias de control y tienen en común el interés por resolver situaciones actuales que generan o pueden generar grandes impactos de salud pública (Backer, van Roermund, Fischer, van Asseldonk \& Bergevoet,2015; Kar \& Jana, 2013; Weidemann, Dehnert, Koch, Wichmann \& Höhle, 2014; Sato, Ito, Sawai \& Iwata, 2015; Aldila, Götz \& Soewono, 2013; Yakob, Riley, Paterson \& Clements, 2013; Rodrigues, Monteiro \& Torres, 2014) o grandes impactos económicos por enfermedades en bovinos, porcinos y aves (Álvarez, Bezos, de la Cruz, Casal, Romero \& Domínguez, 2014; Fischer, van Roermund, Hemerik, van Asseldonk \& de Jong, 2005; Santman-Berends, Mars, van Duijn \& van Schaik, 2015; Van Hulze, Koets, Niele, Heuven, Van Arendonk \& Klinkenberg, 2014).

Tras analizar con mayor profundidad los modelos que buscan controlar situaciones que ponen en riesgo la salud pública, se encuentran diversas propuestas como el modelamiento del comportamiento epidemiológico de la bacteria Clostridium difficile (Yakob, Riley, Paterson \& Clements, 2013), que es la principal causa de diarrea infecciosa en pacientes 
hospitalizados, la cual se describe mediante un modelo estocástico matemático, mostrado en la figura 3, que tiene como objetivo evaluar métodos que permitan controlar y reducir la propagación de la enfermedad.

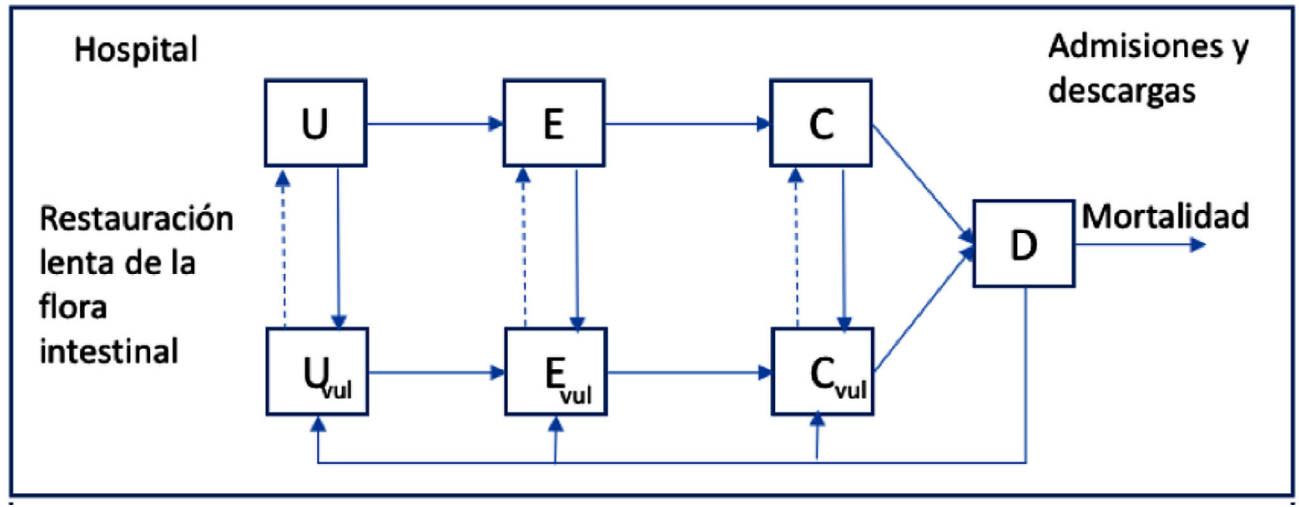

Figura 3. Modelo epidemiológico para la bacteria Clostridium difficile.

Fuente: Tomada de (Yakob, Riley, Paterson \& Clements, 2013).

El modelo de la figura 3 cuenta con los estados U (Unexposes - no expuesto), E (Exposed - expuesto), C (Colonized - colonizado por la bacteria), D (Diseased - enfermo), adicionalmente se establecen los estados finalizados en "Vul", que corresponden a sus pares, pero aplican para personas que han consumido medicamentos antimicrobiales; las flechas negras de la parte superior derecha describen el ingreso y salida de pacientes al hospital, las transiciones en línea punteada se derivan de una restauración de la flora intestinal y las transiciones continuas son producto del cambio de estado del paciente. Como conclusiones del estudio se determinan la ineficacia de la aplicación de medicamentos y de prebióticos para disminuir la incidencia de la enfermedad, sin embargo, los cuidados de las condiciones de higiene tienen un efecto significativo en la prevalencia de la enfermedad.

El segundo enfoque observado en los artículos que usan nuevos modelos para evaluar estrategias de control es la inclusión de factores económicos, ya sea en el propio modelo o como criterio de evaluación de las estrategias de control o de herramientas de toma de decisiones, en este enfoque se determinan los efectos de implementar un programa de control nacional para la Diarrea Viral Bovina DVB en los países bajos (Santman-Berends, Mars, van Duijn \& van Schaik, 2015), proponiéndose un modelo epidemiológico tipo SVIR que permite determinar la prevalencia de la DVB y posteriormente la salida del modelo epidemiológico sirve como entrada de un modelo estocástico obteniendo el costo total del programa de control de la enfermedad. El resultado se muestra en la figura 4 donde se describen varios escenarios, algunos que tienen un costo mayor a la situación actual y otros donde gracias a estrategias como la erradicación voluntaria o el uso de marcas en las orejas de los animales para realizar seguimiento, se logra reducir el costo total producto de la diarrea Bovina. 


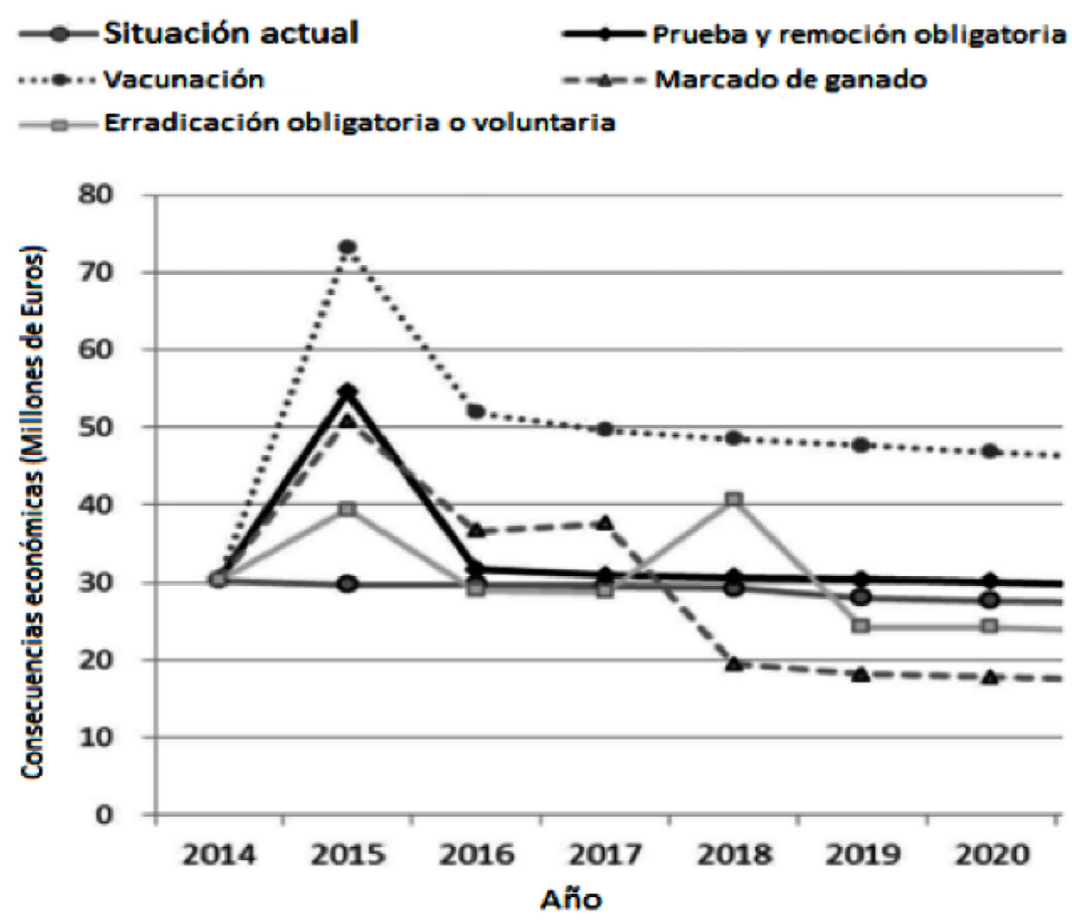

Figura 4. Proyección de costos de la implementación de un programa de control de la diarrea bovina en Holanda.

Fuente: Tomado de (Santman-Berends, Mars, van Duijn \& van Schaik, 2015).

\section{Uso de Modelos Existentes}

\section{para el Análisis de Enfermedades}

La utilización de modelos epidemiológicos previos para analizar enfermedades es una tendencia importante en las publicaciones recientes representada por $19.23 \%$ de los artículos consultados (figura 2), ya que esta categoría deja a un lado las estrategias de control. En general, las investigaciones buscan ajustar y adaptar modelos y enfermedades ya caracterizadas con las condiciones de lugares específicos o razas determinadas, enfoque bajo el cual se desarrollan otras investigaciones (Kang, Gunaseelan \& Abbas, 2014; Kang, Gunaseelan \& Abbas, 2015; Pittavin, Ferreri, Giacobini, Bertolotti, Rosati \& Venturino, 2014). Por otra parte, se busca identificar factores que aun cuando no tengan vacuna están afectando situaciones susceptibles de ser descritas mediante modelos epidemiológicos (Savall, Bidot, Leblanc-Maridor, Belloc \& Touzeau, 2016; Morris, 2015; Milne, Gunn, Entrican \& Longbottom, 2009; Cortés, Sánchez-Sánchez, Santonja \& Villanueva, 2013; Holko, M $\square$ drek, Pastuszak \& Phusavat, 20136). En resumen, esta tendencia permite mejorar la comprensión de las enfermedades epidémicas.

En el artículo descrito por Kang, Gunaseelan \& Abbas (2014), se observa la primera tendencia, quienes mediante un modelo SIR clásico representan la brucelosis bovina, pero utilizando parámetros ajustados a India, la figura 5 muestra el modelo utilizado por el autor y en la tabla 2 se muestran los parámetros propios de India para el ajuste del modelo epidemiológico. 


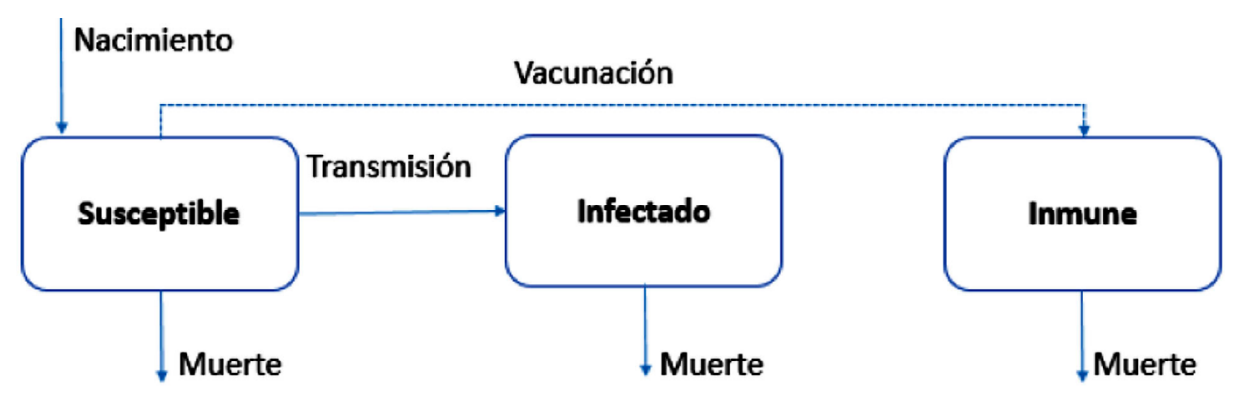

Figura 5. Modelo SIR que describe la Brucelosis bovina.

Fuente: Tomado de (Vynnycky \& White, 2010).

Tabla 2. Parámetros modelo SIR brucelosis india.

\begin{tabular}{l|c} 
Parámetro & Valor \\
\hline $\begin{array}{l}\text { Proporción susceptible }(S)- \\
\text { estabilidad endémica }\end{array}$ & 0.865 \\
\hline $\begin{array}{l}\text { Proporción Infectada }(I)- \\
\text { estabilidad endémica }\end{array}$ & 0.135 \\
\hline Tasa de transmisión $(\beta)$ & $0.1156 /$ year \\
\hline Tasa de Nacimiento/Muerte $(\mu)$ & $0.1 /$ year \\
\hline Eficacia de la vacuna $(v)$ & $70 \%$ \\
\hline Proporción de vacunados $(p)$ & $0-100 \%$ \\
\hline
\end{tabular}

Fuente: Tomado de (Kang, Gunaseelan \& Abbas, 2014).

Por otra parte, se encuentran investigaciones publicadas en las que se usan los modelos previamente descritos para identificar factores que afectan el comportamiento de una enfermedad o situación susceptible de ser modelada epidemiológicamente y este resultado se observa muy bien en el artículo de Milne, Gunn, Entrican \& Longbottom (2009), quienes utilizan dos modelos epidemiológicos tipo SEIR y SIR para describir el comportamiento del aborto en ovejas por clamidia y de esta manera, identificar parámetros claves que impactan la enfermedad como las tasas de contacto entre animales y con material infectado. Producto de esta investigación se determinó que la identificación de ovejas enfermas antes del parto disminuye drásticamente la tasa de abortos, pese a que la forma en la que se contagia la enfermedad no es comprendida claramente.

\section{Análisis de Enfermedades y Epidemias Mediante Nuevos Modelos.}

Esta categoría abarca el $50 \%$ de las publicaciones revisadas (figura 2) siendo la más recurrente, aquí se encuentran las publicaciones que hacen uso de nuevas técnicas y herramientas para ampliar las posibilidades de los modelos epidemiológicos. Es común ver que los modelos epidemiológicos se enriquecen con componentes aleatorios y probabilísticos (Casabán, Cortés, Navarro-Quiles, Romero, Roselló \& Villanueva, 2016; Casabán, Corté, Romero \& Roselló, 2015), donde los parámetros que definen modelos SIS y SI (susceptible - infectado) son de carácter aleatorio.

También se identifican investigaciones donde el uso de variables discretas mejora el desempeño de los modelos planteados (Cauchemez \& Ferguson, 2008); otras donde se hace uso de técnicas matemáticas como perturbación singular (Ssematimba, Jores \& Mariner, 2015), métodos numéricos (Öztürk \& Gülsu, 2015), inyección de ruido blanco (Zhang, Jiang, Liu \& O’Regan, 2016) o modelos bayesianos (Ligarte, Goicoa, Ibáñez \& Militino, 2009). 
Si bien, los modelos epidemiológicos representan una gran herramienta para el análisis de enfermedades y epidemias, en publicaciones como Rist, Ngonghala, Garchitorena, Brook, Ramananjato, Miller, et al. (2015) donde se evalúan factores económicos de Madagascar para modelar enfermedades de aves de corral que afectan la población pobre, o en Sieber, Malchow \& Hilker (2014) donde se formula un modelo epidemio-ecológico que permite evaluar cómo el cambio de comportamiento producido por enfermedades infecciosas en los individuos de un sistema cazador-presa modifica tremendamente la estabilidad del mismo o el enfoque espacial expuesto en Staubach, Schmid, Knorr-Held \& Ziller, (2002); Binder, Ross \& Simpson, (2012) y Daniel, Hengartner, Rivera, Powell \& McPherson (2013) demuestran que el utilizar modelos que combinan elementos epidemiológicos y elementos provenientes de otras áreas del conocimiento fortalecen el área de investigación y abren la posibilidad de generar nuevo y más diverso conocimiento.

\section{ANÁLISIS}

Cuando es necesario elegir un modelo epidemiológico para modelar una enfermedad infecciosa o cualquier otro fenómeno que sea susceptible de modelarse mediante esta técnica, es de vital importancia identificar las características particulares del fenómeno, el entorno en el que se desarrolla y la finalidad del modelo; dicho análisis junto con el conocimiento del área de estudio, permiten determinar si es posible usar o ajustar un modelo ya existente o es mejor plantear un modelo nuevo.

Tras analizar el enfoque de los artículos consultados (Figura 3) se observa una mayor tendencia por el uso de nuevos modelos, si bien, el plantear nuevos modelos que incorporan técnicas y teorías que previamente no hacían parte del modelado de epidemias, enriquece el área del conocimiento y abre las puertas a nuevas posibilidades, por lo general se hace a nivel analítico, como herramienta para entender la dinámica del fenómeno y la efectividad del modelo epidemiológico. Cuando el objetivo es tomar decisiones que pueden significar vidas, los autores son más conservadores, pues usan modelos existentes que ya hayan sido probados en diferentes escenarios y analizados por otros pares, tal es el caso expuesto por Atkins, Shim, Pitzer \& Galvani (2012) quienes usan un modelo previamente planteado y bien sustentado para la toma de decisiones con respecto a la vacunación contra el rotavirus en Gales.

En el caso de estrategias de control con el uso de modelos nuevos, si bien los autores agregan nuevas herramientas y enfoques, estos parten de la solidez de modelos bien fundamentados y agregan elementos que permitan mejorar el desempeño del modelo o se aproximen más al fenómeno real, de esta forma se obtiene la confiabilidad y aceptación del modelo previo, necesarias para tomar decisiones de vida o muerte, junto con las nuevas posibilidades que ofrecen la inclusión de nuevos elementos al modelo.

\section{CONCLUSIONES}

La utilidad y enfoque que pueden tener los modelos epidemiológicos de enfermedades infecciosas es muy amplio, sin embargo, en el presente documento luego de describir las generalidades de los modelos epidemiológicos de enfermedades infecciosas se logra caracterizar los planteamientos descritos en la literatura revisada de acuerdo con 2 variables, el tipo de modelo utilizado (nuevo o ya existente) y la finalidad del modelo (evaluar estrategias de control o analizar e identificar características de enfermedades). Sin embargo, cada documento puede abarcar diversas temáticas y mezclar en mayor o menor medida características de cada una de las categorías creadas. La lucha contra las enfermedades es continua y a medida que surgen nuevas herramientas técnicas y 
tecnológicas será posible modelar fenómenos infecciosos que en la actualidad no es posible, que se modelan de forma parcial o que surjan en el futuro afortunadamente este es un campo de la ciencia de gran interés en la actualidad que recibe los aportes muchas personas.

\section{AGRADECIMIENTOS}

Este trabajo contó con el apoyo de los integrantes del Grupo de Investigación en Medicina Veterinaria y Zootecnia - GIDIMEVETZ, de la Universidad Pedagógica y Tecnológica de Colombia, profesionales que entregaron información y así mismo validaron los procesos en cada etapa de desarrollo del proyecto.

\section{LITERATURA CITADA}

Aldila D., Götz T. \& Soewono E. (2013). An optimal control problem arising from a dengue disease transmission model. Mathematical Biosciences; 242(1): 9-16. http://doi.org/http://dx.doi. org/10.1016/j.mbs.2012.11.014

Álvarez J., Bezos J., de la Cruz M.L., Casal C., Romero B., Domínguez L., et al. (2014). Bovine tuberculosis: Within-herd transmission models to support and direct the decision-making process. Res Vet Sci; 97, Supple, S61 - S68. http://doi. org/http://dx.doi.org/10.1016/j.rvsc.2014.04.00

Atkins K.E., Shim E., Pitzer V.E. \& Galvani A.P. (2012). Impact of rotavirus vaccination on epidemiological dynamics in England and Wales. Vaccine; 30(3): 552-564. http://doi.org/http:// dx.doi.org/10.1016/j.vaccine.2011.11.064

Bacaër N. (2011). A Short History of Mathematical Population Dynamics. New York, Springer. http:// doi.org/10.1007/978-0-85729-115-8

Backer J.A., van Roermund H.J., Fischer E.A., van Asseldonk M.A. \& Bergevoet, R.H. ()2015. Controlling highly pathogenic avian influenza outbreaks: An epidemiological and economic model analysis. Prev Vet Med; 121(1-2): 142-150. http://doi.org/http://dx.doi.org/10.1016/j.prevetmed.2015.06.006

Bekara M.A., Courcoul A., Bénet J. \& Durand B. (2014). Modeling tuberculosis dynamics, detection and control in cattle herds. PloS One; 9(9), e108584. http://doi.org/10.1371/journal.pone.0108584
Binder B.J., Ross J.V. \& Simpson M.J. (2012). A hybrid model for studying spatial aspects of infectious diseases. ANZIAM Journal; 54(1-2): 37-49. http://doi.org/10.1017/S1446181112000296Cai Y., Wang Z. \& Wang W. (2016). Endemic dynamics in a host-parasite epidemiological model within spatially heterogeneous environment. Applied Mathematics Letters; 61: 129-136. http://doi. org/http://dx.doi.org/10.1016/j.aml.2016.05.011

Casabán M., Cortés J., Navarro-Quiles A., Romero J., Roselló M. \& Villanueva R. (2016). A comprehensive probabilistic solution of random SIStype epidemiological models using the random variable transformation technique. Communications in Nonlinear Science and Numerical Simulation; 32: 199-210. http://doi.org/http://dx.doi. org/10.1016/j.cnsns.2015.08.009

Casabán M., Cortés J., Romero J. \& Roselló M. (2015). Probabilistic solution of random SI-type epidemiological models using the Random Variable Transformation technique. Communications in Nonlinear Science and Numerical Simulation; 24(1-3): 86-97. http://doi.org/http://dx.doi. org/10.1016/j.cnsns.2014.12.016

Cauchemez S. \& Ferguson N.M. (2008). Likelihoodbased estimation of continuous-time epidemic models from time-series data: Application to measles transmission in London. Journal of the Royal Society Interface; 5(25): 885-897. http:// doi.org/10.1098/rsif.2007.1292

Cortés J., Sánchez-Sánchez A., Santonja F. \& Villanueva R.J. (2013). Non-parametric probabilistic forecasting of academic performance in Spanish high school using an epidemiological modelling approach. Applied Mathematics and Computation; 221: 648-661. http://doi.org/http://dx.doi. org/10.1016/j.amc.2013.06.070

Daniel W.B., Hengartner N.W., Rivera M.K., Powell D.R. \& McPherson T.N. (2013). An epidemiological model of spatial coupling for trips longer than the infectious period. Mathematical Biosciences; 242(1): 1-8. http://doi.org/http://dx.doi. org/10.1016/j.mbs.2012.11.002

Fan K., Zhang Y. \& Gao S. (2016). On a new ecoepidemiological model for migratory birds with modified Leslie-Gower functional schemes. Advances in Difference Equations; 97(1). http://doi. org/10.1186/s13662-016-0825-3

Fischer E.A.J., van Roermund H.J.W., Hemerik L., van Asseldonk M.A.P.M. \& de Jong M.C.M. (2005). Evaluation of surveillance strategies for 
bovine tuberculosis (Mycobacterium bovis) using an individual based epidemiological model. Prev Vet Med; 67(4): 283-301. http://doi.org/http:// dx.doi.org/10.1016/j.prevetmed.2004.12.002

Gaucel S., Laroche B., Ezanno P., Vergu E. \& Touzeau S. (2009). Using singular perturbations to reduce an epidemiological model: Application to bovine viral diarrhoea virus within-herd spread. J Theor Biol; 258(3): 426-436. http://doi.org/ http://dx.doi.org/10.1016/j.jtbi.2008.08.011

Hernández M. (2007). Epidemiología: diseño y análisis de estudios. 2nd ed. Editorial Médica Panamericana.

Holko A., Mẹdrek M., Pastuszak Z. \& Phusavat K. (2016). Epidemiological modeling with a population density map-based cellular automata simulation system. Expert Systems with Applications; 48: 1-8. http://doi.org/http://dx.doi. org/10.1016/j.eswa.2015.08.018

Kang G.J., Gunaseelan L. \& Abbas K.M. (2014). Epidemiological modeling of bovine brucellosis in India. In Big Data (Big Data), IEEE International Conference on 2014; 6-10. http://doi. org/10.1109/BigData.2014.7004420

Kang G., Gunaseelan L. \& Abbas K. (2015). Epidemiological dynamics of bovine brucellosis in India. Annals of Global Health; 81(1): 127128. http://doi.org/http://dx.doi.org/10.1016/j. aogh.2015.02.793

Kar T.K. \& Jana S. (2013). A theoretical study on mathematical modelling of an infectious disease with application of optimal control. Biosystems; 111(1): 37-50. http://doi.org/http://dx.doi. org/10.1016/j.biosystems.2012.10.003

Keeling M. J. \& Rohani P. (2011). Modeling infectious diseases in Humans and Animals. New Jersey, USA: Princeton University Press. http:// www.scopus.com/inward/record.url?eid=2-s2.0 84884049994\&partnerID $=40 \& m d 5=c 999 c 61563 d$ e33ed4e8a6171c364ad25. Consultado 20 Jul, 2017.

Kermack W.O. \& McKendrick A.G. (1927). A Contribution to the Mathematical Theory of Epidemics. Proceedings of the Royal Society of London A: Mathematical, Physical and Engineering Sciences; 115(772): 700-721. http://doi.org/10.1098/ rspa.1927.0118

Ligarte M.D., Goicoa T., Ibáñez B. \& Militino, A.F. (2009). Evaluating the performance of spatiotemporal Bayesian models in disease mapping. Environmetrics; 20(6): 647-665. http://doi. org/10.1002/env.969
Marutschke D.M. \& Ogawa H. (2014). Clustering Scientific Publication Trends in Cultural Context Using Epidemiological Model Parameters. Procedia Technology; 18: 90-95. http://doi.org/http:// dx.doi.org/10.1016/j.protcy.2014.11.018

Milne C.E., Gunn G.J., Entrican G. \& Longbottom D. (2009). Epidemiological modelling of chlamydial abortion in sheep flocks. Vet Microbiol; 135(1-2): 128-133. http://doi.org/http://dx.doi. org/10.1016/j.vetmic.2008.09.032

Morris R.S. (2015). Diseases, dilemmas, decisionsConverting epidemiological dilemmas into successful disease control decisions. Prev Vet Med; 122(1-2): 242-252. http://doi.org/http://dx.doi. org/10.1016/j.prevetmed.2015.05.003

Njagarah J.B. \& Nyabadza F. (2014). A metapopulation model for cholera transmission dynamics between communities linked by migration. Applied Mathematics and Computation; 241: 317331. http://doi.org/http://dx.doi.org/10.1016/j. amc.2014.05.036

Öztürk Y. \& Gülsu M. (2015). Numerical solution of a modified epidemiological model for computer viruses. Applied Mathematical Modelling; 39(2324): 7600-7610. http://doi.org/http://dx.doi. org/10.1016/j.apm.2015.03.023

Pedersen S. (2015). Algunos Modelos matemáticos para (algunas) enfermedades contagiosas: transmisión, infección, tratamiento [Tesis de Licenciatura]. Buenos Aires, Universidad de Buenos Aires.

Pérez J. M. (2010). Inteligencia computacional inspirada en la vida. SPICUM - Universidad de Málaga.

Pittavino M., Ferreri L., Giacobini M., Bertolotti L., Rosati S. \& Venturino E. (2014). A CAEV epidemiological model for goat breeding. Applied Mathematics and Computation; 227: 156-163. http://doi.org/http://dx.doi.org/10.1016/j. amc.2013.11.030

Rao D.M. (2016). Efficient parallel simulation of spatially-explicit agent-based epidemiological models. Journal of Parallel and Distributed Computing; 93-94: 102-119. http://doi.org/http:// dx.doi.org/10.1016/j.jpdc.2016.04.004

Rist C.L., Ngonghala C.N., Garchitorena A., Brook C.E., Ramananjato R., Miller A.C., et al. (2015). Modeling the burden of poultry disease on the rural poor in Madagascar. One Health; 1: 60-65. http://doi.org/http://dx.doi.org/10.1016/j.onehlt.2015.10.002 
Rodrigues H.S., Monteiro M.T.T. \& Torres D.F.M. (2014). Vaccination models and optimal control strategies to dengue. Mathematical Biosciences; 247: 1-12. http://doi.org/http://dx.doi. org/10.1016/j.mbs.2013.10.006

Rossi G., De Leo G.A., Pongolini S., Natalini S., Vincenzi S. \& Bolzoni L. (2015). Epidemiological modelling for the assessment of bovine tuberculosis surveillance in the dairy farm network in EmiliaRomagna (Italy). Epidemics; 11: 62-70. http:// doi.org/10.1016/j.epidem.2015.02.007

Sahneh F.D., Vajdi A., Melander J. \& Scoglio C.M. (2017). Contact Adaption during Epidemics: A Multilayer Network Formulation Approach. IEEE Transaction on Network Science and Engineering. DOI 10.1109/TNSE.2017.2770091

Santman-Berends I.M.G.A., Mars M.H., van Duijn L. \& van Schaik G. (2015). Evaluation of the epidemiological and economic consequences of control scenarios for bovine viral diarrhea virus in dairy herds. J Dairy Sci; 98(11): 7699-7716. http:// doi.org/http://dx.doi.org/10.3168/jds.2014-9255

Sato A.H., Ito I., Sawai H. \& Iwata K. (2015). An epidemic simulation with a delayed stochastic SIR model based on international socioeconomic-technological databases. Big Data (Big Data), 2015 IEEE International Conference on: 2732-2741. http://doi.org/10.1109/BigData.2015.7364074

Savall J.F., Bidot C., Leblanc-Maridor M., Belloc C. \& Touzeau S. (2016). Modelling Salmonella transmission among pigs from farm to slaughterhouse: Interplay between management variability and epidemiological uncertainty. Int $\mathrm{J}$ Food Microbiol; 229: 33-43. http://doi.org/http://dx.doi. org/10.1016/j.ijfoodmicro.2016.03.020

Sieber M., Malchow H. \& Hilker F.M. (2014). Disease-induced modification of prey competition in eco-epidemiological models. Ecological Complexity; 18: 74-82. http://doi.org/10.1016/j.ecocom.2013.06.002

Ssematimba A., Jores J. \& Mariner J.C. (2015). Mathematical modelling of the transmission dynamics of contagious bovine pleuropneumonia reveals minimal target profiles for improved vaccines and diagnostic assays. PLOS ONE 2015; 10(2). http://doi.org/10.1371/journal. pone. 0116730

Staubach C., Schmid V., Knorr-Held L. \& Ziller M. (2002). A Bayesian model for spatial wildlife disease prevalence data. Prev Vet Med;
56(1): 75-87. http://doi.org/10.1016/S01675877(02)00125-3

University of Chicago. (1989). Hipócrates. Hippocratic writings. On airs, waters and places. http:// classics.mit.edu/Hippocrates/airwatpl.2.2.html. Consultado 23 Jul, 2017.

Van Effelterre T., Marano C. \& Jacobsen K.H. (2016). Modeling the hepatitis A epidemiological transition in Thailand. Vaccine; 34(4): 555-562. http://doi.org/http://dx.doi.org/10.1016/j.vaccine.2015.11.052

Van Hulzen K.J.E., Koets A.P., Nielen M., Heuven H.C.M., Van Arendonk J.A.M.\& Klinkenberg D. (2014). The effect of genetic selection for Johne's disease resistance in dairy cattle: Results of a genetic-epidemiological model. J Dairy Sci; 97(3): 1762-1773. http://doi.org/10.3168/ jds.2013-7032

Vynnycky E. \& White R. (2010). An Introduction to Infectious Disease Modelling. 1nd ed. OUP Oxford.

Weidemann F., Dehnert M., Koch J., Wichmann O. \& Höhle M. (2014). Modelling the epidemiological impact of rotavirus vaccination in Germany - A Bayesian approach. Vaccine; 32(40): 5250-5257. http://doi.org/http://dx.doi.org/10.1016/j.vaccine.2014.06.090

Yakob L., Riley T.V., Paterson D.L. \& Clements A.C.A. (2013). Clostridium difficile exposure as an insidious source of infection in healthcare settings: An epidemiological model. BMC Infectious Diseases; 13(1). http://doi.org/10.1186/14712334-13-376

Zaman G., Kang Y.H. \& Jung I.H. (2009). Optimal treatment of an SIR epidemic model with time delay. Biosystems; 98(1): 43-50. http:// doi.org/http://dx.doi.org/10.1016/j.biosystems.2009.05.006

Zhang Q., Jiang D., Liu Z., O’Regan D. (2016). Asymptotic behavior of a three species eco-epidemiological model perturbed by white noise. Journal of Mathematical Analysis and Applications; 433(1): 121-148. http://doi.org/http://dx.doi. org/10.1016/j.jmaa.2015.07.025 
Félix Sebastián Rincón Tobo, Javier Antonio Ballesteros-Ricaurte, Ángela María González Amarillo

Analizando la evolución del modelado de enfermedades infecciosas

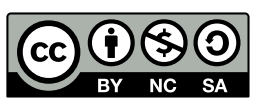

Licencia de Creative Commons

Revista de Investigación Agraria y Ambiental is licensed under a Creative Commons

Reconocimiento-NoComercial-CompartirIgual 4.0 Internacional License. 